\title{
Influência do tempo de menopausa na qualidade de vida e sua relação com a migrânea
}

\author{
Influence of time of menopause in quality of life and its relation \\ to migraine
}

\begin{abstract}
Sidraiton Sálvio Alves de Melo Filho', Marcelo Moraes Valença²
'Ginecologista, Mestrando em Neurociências pela Pós-graduação em Neuropsiquiatria e Ciências do Comportamento do Centro de Ciências da Saúde da Universidade Federal de Pernambuco - UFPE, Recife, PE, Brasil

${ }^{2}$ Livre-Docente em Neurocirurgia, Faculdade de Medicina de Ribeirão Preto,

Universidade de São Paulo - FMRP-USP; Professor Associado de Neurologia e Neurocirurgia, Departamento de Neuropsiquiatria da UFPE; Coordenador da Pós-Graduação em Neuropsiquiatria, UFPE

Melo Filho SS, Valença MM

Influência do tempo de menopausa na qualidade de vida e sua relação com a migrânea. Headache Medicine. 2012;3(1):13-20
\end{abstract}

\begin{abstract}
RESUMO
O tempo decorrido após a menopausa influencia os sintomas do hipoestrogenismo experimentados pelas mulheres, e as características dos ataques de migrânea naquelas acometidas pela doença. Esta revisão buscou evidências científicas alusivas à relação entre qualidade de vida $(Q V)$ e as fases da pós-menopausa e à influência das oscilações hormonais do climatério nas mulheres com migrânea. Foram pesquisados os termos quality of life, menopause, perimenopause, early menopause, late menoapause, climacteric symptoms, hormonal, estrogens, migraine, pathophysiology nas bases MEDLINE (PubMed) e SciELO Brasil. Tornou-se clara a relação dos estrogênios com a fisiopatogenia da migrânea e esta pode ser a chave para explicar possíveis diferenças na QV entre as fases da pós-menopausa. Não existem estudos observacionais para avaliar possíveis diferenças na QV entre as fases da pós-menopausa e averiguar o papel da migrânea com as eventuais diferenças encontradas.
\end{abstract}

Palavras-chave: Qualidade de vida; Menopausa; Climatério; Estrogênios; Migrânea; Pós-menopausa recente; Pósmenopausa tardia

\section{ABSTRACT}

The time since menopause influences the hypoestrogenism symptoms experienced by women and characteristics of migraine attacks in those with the disease. This review aimed to scientific evidence alluding to the relationship between quality of life (QOL) and the phases of post-menopause and the influence of hormonal fluctuations of menopause in women with migraine. The terms quality of life, menopause, perimenopause, early menopause, late menopause, climacteric symptoms, hormones, estrogens, migraine, pathophysiology have been searched in MEDLINE (PubMed) and SciELO Brasil. It became clear the relationship of estrogens to the pathophysiology of migraine and this may be the key to explain possible differences in QOL between the phases of postmenopause. Lack of observational studies to evaluate possible differences in QOL between the phases of post-menopause and examine the role of migraine with any differences.

Keywords: Quality of life; Menopause; Estrogens; Migraine; Early menopause; Late menopause

\section{INTRODUÇÃO}

A pós-menopausa é dividida em recente (os primeiros cinco anos de menopausa) e tardia (os dez anos seguintes). Na perimenopausa e pós-menopausa recente, prevalecem os sintomas vasomotores (fogachos, sudorese fria), tendência ao humor depressivo e piora da intensidade e na frequência das crises em pacientes com migrânea. Na pós-menopausa tardia prevalecem sintomas físicos (fadiga, dores osteoarticulares, etc.) e ligados à atrofia tecidual (urogenital, da pele e das mamas, entre 
outros). Relatos na literatura sugerem a relação entre qualidade de vida (QV) e tempo de menopausa. ${ }^{(1-3)}$ Vários estudos demostraram o impacto da migrânea na qualidade de vida, levando a Organização Mundial de Saúde (OMS) a colocar essa doença na posição de número 19 na lista de doenças mais incapacitantes em todo o mundo. ${ }^{(4)}$ Faz-se então necessário conhecermos de que forma a QV se altera no decorrer das fases da menopausa e o papel da migrânea nesta alteração.

\section{MÉTODOS}

O presente estudo é uma revisão de literatura. A metodologia consistiu na procura de evidências científicas alusivas à relação entre QV e as etapas da pós-menopausa, bem como à influência das oscilações hormonais do climatério nas mulheres com migrânea. Para tal, efetuou-se pesquisas nas bases de dados eletrônicas MEDLINE (PubMed) e SciELO Brasil, ligadas ao Portal CAPES.

Utilizaram-se os seguintes termos para pesquisa: quality of life, menopause, perimenopause, early menopause, late menopause, climacteric symptoms, hormonal, estrogens, migraine, pathophysiology. Os referidos termos foram pesquisados isolados ou associados. Atendendo à finalidade de revisão da literatura científica existente, não se delimitou qualquer período para a publicação de artigos. Excluíram-se aqueles que não se apresentavam nas línguas inglesa ou portuguesa. Para cada citação relevante, a bibliografia foi revista a fim de identificar fontes adicionais de dados pertinentes.

\section{Climatério}

Climatério é o período de vida da mulher compreendido entre o fim da fase reprodutiva e o início da senilidade ou senectude. O termo climatério é originado do grego klimacton, que significa crise ou período crítico. A idéia de "crise" que se tinha na Grécia antiga pode se justificar pelas intensas mudanças que se observam em todo o corpo feminino, neste período da vida, consequentes à queda da produção de estrogênios pelos ovários. Há atrofia progressiva da pele e seus anexos e também dos órgãos genitais, urinários e das mamas. Alterações do metabolismo dos lipídeos e dos ossos são uma regra, havendo maior tendência à obesidade, à fadiga e ao aumento da incidência de doenças cardiovasculares e osteoporose. Diminuição da acuidade visual (presbiopia) também é observada. ${ }^{(5)}$ No sistema nervoso central (SNC) há alterações sobre os centros termorreguladores hipotalâmicos e, como consequência, se tornam comuns sintomas vasomotores, como ondas de calor e sudorese. Nos últimos anos, também se tem relacionado o hipoestrogenismo a modificações neurológicas, entre elas à doença de Alzheimer. ${ }^{(6-8)}$ Outros efeitos dos estrogênios sobre o SNC justificam a relação destes hormônios com migrânea. ${ }^{(9)}$

A diminuição dos níveis estrogênicos é progressiva e quando estes não apresentam concentrações suficientes para desencadear a liberação do pico pré-ovulatório do hormônio luteinizante, surgem os ciclos anovulatórios e irregularidades menstruais, de modo que as menstruações ficam mais espaçadas. Quando a mulher climatérica completa doze meses de amenorreia, diz-se que a mesma atingiu a menopausa, que é o advento da última menstruação. Segundo a Sociedade Brasileira de Climatério (SOBRAC), podemos dividir o climatério em três fases: ${ }^{(10)}$

- Pré-menopausa: vai do final da vida reprodutiva até o momento da menopausa

- Perimenopausa: abrange o duplo período de dois anos, que precede e sucede a menopausa

- Pós-menopausa: inicia-se na menopausa e finda na senectude. Esta, por sua vez, se divide em:

- Pós-menopausa recente: são os primeiros cinco anos após a menopausa.

- Pós-menopausa tardia: são os dez anos seguintes ao término da pós-menopausa recente.

Com o fim da menopausa tardia, isto é, após quinze anos do advento da última menstruação, segundo a SOBRAC, a mulher entra numa outra etapa da vida chamada de senectude ou senilidade.

A síndrome do climatério, moléstia menopausal ou síndrome menopausal compreende conjunto de sintomas e sinais que aparecem no climatério, prejudicando o bemestar da mulher.

\section{Qualidade de vida no climatério}

Para a OMS, QV é a percepção que uma pessoa tem de sua posição na vida, no contexto do sistema de valores e da cultura a que está inserida, em relação às suas metas, expectativas, padrões e crenças. ${ }^{111}$ Percebe-se que QV tem um conceito amplo e multidimensional, visto que não só envolve os aspectos relacionados à saúde física e mental do indivíduo, mas também aspectos outros, de cunho emocional, econômico, social, cultural e espiritual. $O$ conceito de QV é também subjetivo, ao passo em que busca captar qual o ponto-de-vista do indivíduo a respeito de sua própria vida. Em sendo um parâmetro subjetivo por definição, o questionamento direto é o modo mais simples e apropriado para se obterem 
informações a respeito dos sintomas e sensações dos indivíduos em questão. ${ }^{(12)}$ Foi com o objetivo de transformar conceitos individuais em dados objetivos e mensuráveis, passíveis de serem quantificados, analisados e comparados entre populações diferentes, que foram criados diversos questionários de QV. Tais instrumentos possibilitaram a médicos e a pesquisadores transformar informações subjetivas em medidas quantitativas para que possam ser usadas em ensaios clínicos e em estudos econômicos. ${ }^{(13-15)}$

Cada vez mais, na área de saúde, a QV tem sido avaliada para mensurar o impacto dos agravos à saúde no bem-estar das pessoas. Com os avanços tecnológicos em métodos diagnósticos e tratamentos de doenças, houve um aumento da expectativa de vida das pessoas, ${ }^{(16)}$ mas esse fato não determina que elas tenham uma melhor QV. Pelos dados do Instituto Brasileiro de Geografia e Estatística, a esperança de vida ao nascer da brasileira no ano de 2010 foi de 77 anos. (16) Por outro lado, a idade da menopausa vem se mantendo constante ao longo do tempo. Se houve mudança, contudo, a história indica que foi mínima. ${ }^{(17,18)}$ Sendo assim, a brasileira passa boa parte de sua vida (aproximadamente um terço dela) em situação de hipoestrogenismo, convivendo com os sintomas dele decorrentes.

A avaliação do impacto de uma condição sobre a QV é particularmente relevante em situações sintomáticas, como o climatério. ${ }^{(19,20)}$ Há dados que sugerem uma queda na QV no climatério, tanto na transição menopausal (perimenopausa), quanto nos anos que sucedem o advento da última menstruação. Os aspectos físicos da saúde geral e do bem-estar emocional declinam durante a transição da menopausa. ${ }^{(21,22)}$ Os sintomas climatéricos acometem entre $60 \%$ a $80 \%$ das mulheres e são reconhecidos como indutores de desconforto físico e emocional que aumentam com a gravidade dos sintomas. ${ }^{(19,23)}$ A depender da intensidade e da frequência dos sintomas, em especial as ondas de calor, pode haver interferência no bem-estar e na QV. (24)

Entre os vários questionários de QV utilizados em saúde está o Questionário de Saúde da Mulher (QSM), um dos principais instrumentos de avaliação da QV da mulher climatérica, pesquisando especificamente os problemas relacionados a esta fase da vida. ${ }^{(25)}$ Desenvolvido por Hunter em 1992, para mensurar o relato subjetivo do bem-estar físico e mental de mulheres entre 45 e 65 anos, o QSM foi traduzido e validado para o português do Brasil por Silva-Filho. ${ }^{(26)}$

Seus ítens foram escolhidos com o cuidado de não enfatizarem os sintomas negativos, sendo inclusive cha- mado de Questionário de Saúde (Geral) da Mulher, não incluindo menopausa ou climatério no nome, a fim de que o conhecimento, por parte do sujeito, do propósito de algum estudo de que venha a utilizá-lo, pudesse ser minimizado. ${ }^{(25)}$

O QSM consta de 36 questões a respeito dos sintomas e sensações experimentadas pelos sujeitos, oferecendo quatro alternativas como possibilidade de resposta: 1) Sim, sempre; 2) Sim, algumas vezes; 3) Não, não muito (raramente); 4) Não, nunca.

Suas questões estão divididas em nove domínios, dispostos aleatoriamente, que avaliam:

- Humor depressivo (sete questões) - $3 ; 5 ; 7 ; 8 ; 10$; $12 ; 25 ;$

- Sintomas somáticos (sete questões) - 14;15;16; 18 ; 23; 30; 35;

- Memória/Concentração (três questões) - 20; 33; 36;

- Sintomas vasomotores (duas questões) - 19; 27;

- Ansiedade/temores (quatro questões) - 2; 4;6;9;

- Comportamento sexual (três questões) - 24; $31 ; 34$;

- Distúrbios do sono (três questões) - 1; 11;29;

- Sintomas menstruais (quatro questões) - 17; 22; 26; 28;

- Atratividade (autoestima) (três questões) - 13;21; 32.

O escore do domínio é dado pelo somatório dos pontos de cada sintoma (questão), reduzidos a uma escala binária (Sim, sempre/Sim, algumas vezes $=1$; Não, não muito/Não, nunca $=0$ ), dividido pelo número de sintomas/questões de cada domínio. Sendo assim, cada domínio terá um escore mínimo de 0 e um máximo de 1 , sendo 1 o maior grau de sofrimento ou disfunção apresentado pelo sujeito frente àquele conjunto de sintomas. Para algumas questões a pontuação é invertida, conforme forem indagadas positivamente ou negativamente. As questões cujas pontuações estão invertidas são: $7,10,21,25,31$ e 32 . No caso do sujeito não ser sexualmente ativo, as questões 31 e 34 do domínio comportamento sexual devem ser desconsideradas.

As questões específicas sobre transtornos de humor foram retiradas da escala de Leeds para ansiedade e depressão, ${ }^{(27)}$ que tem grande semelhança com a escala de Hamilton para depressão. (28,29)

Por não se tratar de um questionário discriminativo, não faz diagnósticos específicos, mas possibilita o acesso a informações subjetivas. Em décadas de uso, já foi amplamente utilizado em estudos sobre os sintomas do climatério e mensuração de seu impacto no bem-estar da paciente $^{(30,31)}$ e avaliação da resposta à Terapia Hormonal do Climatério (TH). ${ }^{(32)}$ De todos os sete instrumentos exis- 
tentes com o propósito de avaliar QV na faixa etária do climatério, uma recente revisão sistemática sobre a qualidade de suas propriedades psicométricas, considerou o QSM como o questionário de mais alta pontuação. ${ }^{(33)}$

Recentemente nós conduzimos um estudo descritivo, comparativo e transversal, com mulheres na pós-menopausa. As participantes foram divididas em dois grupos: pós-menopausa recente (com tempo de pós-menopausa menor que 5 anos) e pós-menopausa tardia ( $n=61$, com tempo de pós-menopausa maior ou igual a 5 anos e menor que 15 anos). Avaliando-se o QSM, encontrouse um nível intermediário de QV, não havendo diferença entre os dois grupos $(p>0,05)$. Diferenças significativas foram observadas quando analisados os domínios memória/concentração $(p<0,05)$, sintomas vasomotores $(p<0,05)$ e atratividade $(p<0,05)$, tendo as mulheres da pós-menopausa recente maiores médias dos escores. A prevalência de cefaleia na amostra foi de $58 \%$. Avaliando-se o MIDAS, não se encontrou associação entre o grau de cefaleia e o tempo de menopausa. Porém, houve aumento significativo dos escores QSM com o aumento do grau de impacto da cefaleia no MIDAS para os domínios da QV humor depressivo $(p<0,05)$, sintomas somáticos $(p<0,05)$, sintomas vasomotores $(p<0,05)$, ansiedade/medo $(p<0,05)$ e distúrbios do sono $(p<0,05)$. Assim, não houve diferença na QV global quanto ao tempo de pós-menopausa, porém mulheres na pós-menopausa recente tiveram pior $Q V$ quanto aos sintomas vasomotores, ao déficit cognitivo e à autoestima. No entanto, de forma significativa, quanto mais impacto tinha cefaleia primária, mais frequentes foram os distúrbios do sono e as queixas físicas, vasomotoras e relacionadas à ansiedade e depressão.

\section{Migrânea}

Segundo a Sociedade Internacional de Cefaleia (SIC), a migrânea ou enxaqueca é um tipo de cefaleia primária muito comum e capaz de produzir grande incapacidade ao doente. A sua elevada prevalência tem sido documentada por estudos epidemiológicos, bem como o seu impacto socioeconômico e pessoal. ${ }^{(4)}$ A migrânea pode ser dividida em dois tipos principais:

\section{Migrânea sem aura}

Síndrome clínica caracterizada por cefaleia com características especificas e sintomas associados. É o subtipo mais comum de migrânea, com uma frequência maior de ataques em comparação com o outro subtipo, e usualmente mais incapacitante.
Caracteriza-se por uma cefaleia recorrente, com os seguintes critérios diagnósticos:

A. Mínimo de cinco crises, preenchendo os critérios B-D

B. Crises que duram de 4-72 horas (não tratadas ou tratadas com sucesso)

C. Cefaléia que tem pelo menos duas das seguintes características:

1. Localização unilateral

2. Caráter pulsátil

3. Dor de intensidade moderada ou severa

4. Agravada pelas atividades físicas de rotina, como andar ou subir escadas, ou que causa incapacidade frente a estas atividades.

D. Durante a cefaleia, a presença de pelos menos um dos seguintes sintomas:

1. náusea e/ou vômitos

2. fotofobia e fonofobia

E. Que não seja atribuída a outra patologia.

\section{Migrânea com aura}

Desordem recorrente, que se manifesta em ataques de sintomas neurológicos focais reversíveis (aura), que usualmente surgem gradualmente dentro de 5 a 20 minutos e duram menos de 60 minutos. A aura típica consiste de sintomas visuais e/ou sensoriais e/ou relativos à fala. Cefaleia com as características da migrânea sem aura usualmente segue os sintomas. Menos comumente, a cefaleia não tem as características de migrânea ou está ausente.

Alguns doentes também experimentam uma fase premonitória, antecedendo em horas ou dias o aparecimento da cefaleia e ainda uma fase de resolução da cefaleia. Os sintomas premonitórios e de resolução descritos são hiperatividade, hipoatividade, depressão, apetite para determinados alimentos específicos, bocejos repetidos e outros sintomas inespecíficos relatados por alguns doentes.

\section{Qualidade de vida na migrânea}

A migrânea é um importante problema de saúde pública, causador de grande ônus ao indivíduo e à sociedade. A migrânea é uma das principais causas de internamentos e absenteísmo nos Estados Unidos da América (EUA), refletindo na economia daquele país. A perda de produtividade relativa à migrânea custa aos empregadores americanos cerca de 13 bilhões de dólares por ano. ${ }^{(34)}$ Há também um grande impacto na utilização dos serviços de saúde. O National Ambulatory 
Medical Care Survey, conduzido entre 1976 e 1977, demonstrou que $4 \%$ de todas as visitas aos consultórios de clínicos (cerca de 10 milhões por ano) foram por conta de cefaleia. ${ }^{(35)}$ A migrânea também resulta numa elevada frequência de atendimentos em emergência nos EUA e também no Brasil.(36-38) Uma grande quantidade de medicamentos prescritos e um número ainda maior comprados diretamente no balcão são consumidos pelos americanos todos os anos para tratamento das cefaleias. ${ }^{(36)}$ As vendas de analgésicos comprados diretamente no balcão nos EUA (para todos os tipos de dores) alcançaram a marca de 3,2 bilhões de dólares em 1999 e a cefaleia respondeu por aproximadamente um terço dessas vendas. ${ }^{(39)}$ As vendas de triptanos totalizam cerca de um bilhão de dólares por ano no EUA. (39)

Em 2001, foi estimado que cerca de 28 milhões de cidadãos americanos tinham crises graves de cefaleia. Entre as mulheres americanas com migrânea, 25\% experimentavam quatro ou mais ataques intensos por mês; $48 \%$ experimentavam entre um e quatro ataques intensos por mês e 38\% experimentavam menos que um ataque por mês, ${ }^{(40)}$ padrão semelhante ao que foi visto entre os homens. Também foi demonstrado que $92 \%$ das mulheres e $89 \%$ dos homens com migrânea intensa apresentavam algum grau de invalidez temporária relacionada à cefaleia, e aproximadamente a metade dos casos se apresentava gravemente incapacitada ou com necessidade de repouso acamado. ${ }^{(40)}$ Um grande número de pacientes migranosos com crises incapacitantes vive com medo constante, sabendo que a qualquer momento um ataque poderá comprometer a sua capacidade de trabalhar, de cuidar de si próprio e de seus familiares e de atender a compromissos sociais. Uma vasta quantidade de evidências indica que a migrânea reduz a QV. ${ }^{(41,42)}$ Segundo a OMS, esse agravà a saúde está na posição número 19 dentre todas as doenças que mais causam invalidez em todo o mundo. ${ }^{(4)}$

O questionário MIDAS (Migraine Disability Assessment) foi criado para ajudar os médicos e pacientes na avaliação das cefaleias primárias, em especial para a avaliação da migrânea, para estabelecer uma relação entre esta e a incapacidade por ela provocada. $\bigcirc$ seu principal objetivo foi o de melhorar significativamente o tratamento da migrânea ${ }^{(43)}$ e indiretamente, avaliar o impacto desta doença na QV dos seus portadores.

Os indivíduos com migrânea respondem a cinco questões, atribuindo um valor ao número de dias, nos últimos três meses, em que as suas atividades diárias foram limitadas pela dor. $\bigcirc$ resultado do questionário exprime-se numa pontuação, que se relaciona da seguinte forma com as necessidades de tratamento:

I: Escore 0-5 - Incapacidade mínima ou pouco frequente - necessidade terapêutica muito reduzida ou não necessitam de tratamento.

II: Escore 6-10 - Incapacidade ligeira ou pouco frequente - necessidade moderada de terapêutica.

III: Escore 11-20 - Incapacidade moderada necessidade de terapêutica.

IV: Escore $\geq 21$ - Incapacidade grave - grau III e IV de migrânea, necessidade terapêutica urgente. ${ }^{(43)}$

Nos estudos completos até a data, o MIDAS revelou-se inteiramente consistente, altamente confiável, válido e correlacionado com o julgamento clinico dos médicos. ${ }^{(44)}$ Essas características dão apoio ao uso do MIDAS na pesquisa e na prática clínica. Com o intuito de melhorar a assistência prestada, os portadores de migrânea devem ser encorajados a procurar cuidados médicos e falar sobre a sua doença. Por sua vez, médicos devem realizar o diagnóstico específico, avaliar a incapacidade provocada pela cefaleia e selecionar o tratamento apropriado ao diagnóstico e ao grau de acometimento. O MIDAS, portanto, pode desempenhar um papel importante em iniciativas em saúde pública na abordagem à migrânea.

\section{Migrânea e os estrogênios}

Os estrogênios exercem um efeito de facilitação com os sistemas serotoninérgicos e glutamatérgicos. $\bigcirc$ tônus serotoninérgico nas mulheres está diretamente proporcional aos níveis de estrogênios circulantes. Estudos em animais mostraram que os estrogênios alteram o tônus serotoninérgico. ${ }^{(9)}$ Quando a concentração destes hormônios declina, as concentrações de serotonina acompanham a queda, devido à combinação entre um declínio da sua produção e aumento na sua taxa de excreção. Os estrogênios modulam o metabolismo da serotonina ao afetar a expressão da triptofano-hidroxilase, a enzima limitante da síntese de serotonina, bem como das monoamino-oxidases $A$ e $B$, enzimas responsáveis pela degradação da serotonina. $\bigcirc$ número de transportadores de recaptação de serotonina na fenda sináptica é modificado pela duração à exposição aos estrogênios. Os estrogênios também modulam a sensibilidade dos receptores 5 -HT1 e 5-HT29.

A diminuição resultante da função serotoninérgica está relacionada com a liberação do peptídeo relacionado ao gene da calcitonina e da substância $P$ pelos nervos trigêmeos, que levam à dilatação dos vasos crani- 
anos e sensibilização dos aferentes meníngeos do trigêmeo. ${ }^{(45)}$ Estrogênios também afetam outros mediadores químicos, como o óxido nítrico, o magnésio e prostaglandinas, que têm a capacidade de modular o balanço entre a neurotransmissão excitatória e inibitória. ${ }^{(9,46)}$

Diante disso, não é de se estranhar que crises de migrânea possam ser desencadeadas em mulheres suscetíveis quando há flutuação dos níveis estrogênicos. Pela $2^{a}$ edição da Classificação Internacional das Cefaleias da SIC, a migrânea menstrual é considerada um subtipo de migrânea sem aura que está relacionada aos esteroides ovarianos. A migrânea menstrual é definida como crises de migrânea que ocorrem nos dias -2 e +3 do ciclo menstrual, considerando o dia +1 como sendo o primeiro dia da menstruação. ${ }^{(4)}$

Acredita-se que nos períodos pré-menstrual e menstrual, fases do ciclo menstrual em que os níveis estrogênicos encontram o nadir, a queda da concentração sérica destes hormônios seja o gatilho para o surgimento das crises álgicas. Mulheres com migrânea menstrual parecem ter um resposta anormal ao declínio fisiológico dos níveis de estradiol. ${ }^{(47)}$ Nelas, a estabilização dos níveis estrogênicos por período prolongado parece proteger contra a migrânea.

Dados epidemiológicos dão conta dessa influência dos estrogênios sobre a migrânea. A prevalência de migrânea em países ocidentais é maior em mulheres do que em homens (18\% versus 6\%). ${ }^{(48)}$ Além disso, em 33\% das mulheres, a migrânea se inicia após a menarca, ${ }^{(49)}$ tende a piorar no primeiro trimestre da gravidez e a melhorar nos últimos dois trimestres. ${ }^{(50)}$ Mais de $60 \%$ das mulheres com migrânea relatam associação das crises com a menstruação. ${ }^{(51)}$ Não há evidências de que os outros tipos de cefaleias primárias (tipo tensional ou em salvas) possam ser influenciados pelos níveis de estrogênios.

\section{Migrânea e climatério}

A migrânea apresenta clara tendência de melhora nas mulheres após os 55 anos de idade, período que, em geral, coincide com o início da pós-menopausa tardia, pelo menos em parte, devido à estabilização dos níveis hormonais. ${ }^{(49,52)}$ Além disso, a prevalência de migrânea é maior durante a transição menopausal (quando há início da falência ovariana) do que durante a pósmenopausa recente, particularmente em mulheres com história de migrânea menstrual. ${ }^{(53)}$ Mesmo em pacientes mais idosos, a cefaleia é ainda mais comum em mulheres do que em homens. As mulheres tendem a apresentar mais crises com auras e, ainda, as crises são mais prolongadas do que em homens. ${ }^{(40,54)}$ Alguns estudos relataram que mulheres submetidas à menopausa cirúrgica experimentavam mais provavelmente uma piora das suas crises de migrânea, em relação às mulheres que tiveram menopausa natural. ${ }^{(55,56)} \bigcirc$ uso de anticoncepcionais orais e da TH podem piorar a migrânea ou mudar suas características. ${ }^{(57,58)} \bigcirc$ Women's Health Study relatou uma prevalência de $11 \%$ de migrânea entre 17.107 profissionais de saúde menopausadas (destas 39\% nunca fizeram uso de $\mathrm{TH}$, enquanto $61 \%$ eram usuárias no momento de TH). (59) Foi relatado um odds ratio para migrânea de 1,42 (95\% IC 1,24 - 1,62) para as usuárias, comparadas às que nunca utilizaram $\mathrm{TH}$, após ajustados os fatores de confusão.

\section{DISCUSSÃO}

É clara a relação que os estrogênios têm com a fisiopatogenia da migrânea. Mudanças no padrão das crises já foram bem documentadas durante períodos da vida da mulher em que há grande oscilação dos níveis estrogênicos, como a gravidez e os extremos da vida reprodutiva - menarca (marco dos primeiros ataques de migrânea em quase um terço das mulheres) e climatério. Este último é uma etapa da vida feminina que deve receber especial atenção daqueles que se dedicam à saúde da mulher e ao estudo das cefaleias. As evidências apontam para um agravamento das crises, em frequência e intensidade, na perimenopausa e pós-menopausa recente, enquanto que, na pós-menopausa tardia, há diminuição da gravidade da doença, dada à progressiva diminuição e estabilização dos níveis estrogênicos (considerando apenas mulheres que não usam TH). No entanto, mesmo em situação de hipoestrogenismo, um percentual ainda considerável das mulheres é acometido pela migrânea $(11 \%$, contra os $18 \%$ de prevalência vista durante os anos de vida reprodutiva). Possivelmente, se considerássemos apenas as mulheres no climatério que não fazem uso de $\mathrm{TH}$, a prevalência de migrânea nesta faixa etária seria menor. Não podemos, todavia, esquecer o considerável número de usuárias deste tipo de terapia em nosso meio, frente aos benefícios já bem estabelecidos da TH do climatério sobre a QV.

Reconhecidamente, nas mulheres que não fazem uso de $\mathrm{TH}$, os sintomas inicialmente causados pelo hipoestrogenismo, como os fogachos e as ondas de sudorese noturna (que provocam insônia, que, por sua vez, leva a sintomas como irritabilidade, impaciência, sonolência durante 
o dia, dificuldade de concentração), quando aliados à ansiedade e tendência ao humor depressivo, típicos da perimenopausa e dos primeiros anos de pós-menopausa podem levar a interferência no bem-estar. De outro lado, na pós-menopausa tardia, os sintomas decorrentes do hipoestrogenismo em longo prazo, como a diminuição da libido e a atrofia da mucosa vaginal e uretral (indutoras de desconforto nas relações sexuais), podem levar ao desinteresse pela atividade sexual. A perda progressiva da densidade mineral óssea, culminando em osteoporose (que quando avançada pode ocasionar fraturas patológicas) e os processos degenerativos articulares e musculares provocando dores no corpo e fadiga são sintomas comuns desta fase.

As evidências dão conta de uma piora da QV no climatério, mas não há dados que apontem se há mudança na QV com o passar dos anos. Caso haja mudança da QV com o tempo de menopausa, é possível que a migrânea possa ser preponderante para isso. Porém, esse aspecto ainda não foi adequadamente avaliado. Tendo em vista o grande impacto provocado pela migrânea na QV e a maior morbidade da doença na transição menopausal e pós-menopausa recente, é possível que, havendo mudança na QV, esta seja pior durante este período. Saber as peculiaridades de cada fase da pós-menopausa, entendendo exatamente o que afeta o bem-estar das mulheres, poderá ajudar a melhorar a assistência a saúde delas.

\section{CONCLUSÃO}

Mesmo após décadas de estudo sobre este tema, ainda há necessidade de trabalhos que possam elucidar alguns pontos ainda nebulosos sobre o climatério e, em especial, sobre a QV neste período. Estudos observacionais poderiam ser implementados com o objetivo de avaliar possíveis diferenças na QV entre as duas fases da pós-menopausa e averiguar se a incapacidade provocada pela migrânea tem relação com as eventuais diferenças encontradas.

\section{REFERÊNCIAS}

1. Jacobs PA, Hyland ME, Ley A. Self-rated menopausal status and quality of life in women aged 40-63 years. Br J Health Psychol. 2000;5:395-411.

2. Li S, Holm K, Gulanick M, Lanuza D. Perimenopause and the quality of life. Clin Nurs Res. 2000;9(1):6-23.

3. Utian WH. Quality of life (QOL) in menopause. Maturitas. 2007;57(1):100-2.
4. Second Edition of the International Classification of Headache Disorders (ICHD - 2) - International Headache Society. 2004 [Internet] Disponível em: http://ihs-classification.org/en/

5. Denard-Toulet A. La Ménopause Effacée. Paris. Robert Laffont S. A., 1975

6. Craig MC, Murphy DG. Alzheimer's disease in women; Best Pract Res Clin Obstet Gynaecol.2009;23 (1):53-61.

7. Henderson VW, Brinton RD. Menopause and Mitochondria: windows into estrogens effects on Alzheimer's disease risk and therapy. Prog Brain Res. 2010;182:77-96.

8. Henderson WW. Action of estrogens in aging brain: dementia and cognitive aging. Biochem Biophys Acta. 2010;1800 (10): 1077-83.

9. Martin VT, Behbehani M. Ovarian hormones and migraine headache: understanding mechanisms and pathogenesis--part I. Headache. 2006; 46:3.

10. Halbe HW, Fonseca AM. Síndrome do Climatério. In: Tratado de Ginecologia, volume 2, São Paulo, Roca, 2000

11. The World Health Organization Quality of Life Assessment (WHOQOL): position paper from the World Health Organization. Soc Sci Med. 1995;41(10): 1403-9.

12. Martins MAD, Nahas EAP, Nahas-Neto J, Uemura G, Buttros DAB, Traiman P. Qualidade de vida em mulheres na pós-menopausa, usuárias e não usuárias de terapia hormonal. Rev Bras Ginecol Obstet. 2009;31 (4): 196-202.

13. Faden R, Leplège A. Assessing quality of life. Moral implications for clinical practice. Med Care. 1992;30(5 Suppl):2. MS $166-$ 75.

14. Fitzpatrick R, Fletcher A, Gore S, Jones D, Spiegelhalter D, Cox D. Quality of life measures in health care. I. Applications and issues in 3. assessment. BMJ. 1992;305(6861):1074-7.

15. Quality of life in social science and medicine. Soc Sci Med. 1995;41(10): 1337-8.

16. Instituto Brasileiro de Geografia e Estatística - Síntese de Indicadores Sociais 2010 - Tabelas Aspectos Demográficos: Tabela 1.4; 2010. [internet] Disponível em: ftp://ftp.ibge.gov.br/ Indicadores_Sociais/Sintese_de_Indicadores_Sociais_2010/tabelas/aspectosdemo.zip

17. Amundsen DW, Diers CJ. The age of the menopause in classical Greece and Rome. Hum Biol. 1970; 42(1):79-86.

18. Amundsen DW, Diers CJ. The age of the menopause in medieval Europe. Hum Biol. 1973; 45(4):605-12.

19. Wiklund I. Methods of assessing the impact of climacteric complaints on quality of life. Maturitas. 1998;29(1):41-50.

20. Zöllner YF, Acquadro C, Schaefer M. Literature review of instruments to assess health-related quality of life during and after menopause. Qual Life Res. 2005; 14(2):309-27.

21. Blümel JE, Castelo-Branco C, Kerrigan N, Cancelo MJ, Blümel B, Haya J, et al. Influences of hormone replacement therapy on postmenopausal women's health perceptions. Menopause. 2003; 10(3):235-40.

22. Mishra GD, Brown WJ, Dobson AJ. Physical and mental health: changes during menopause transition. Qual Life Res. 2003; 12(4):405-12.

23. Hess R, Colvin A, Avis NE, Bromberger JT, Schocken M, Johnston $J M$, et al. The impact of hormone therapy on health-related quality of life: longitudinal results from the Study of Women's Health Across the Nation. Menopause. 2008; 15(3):422-8. 
24. van der Mooren MJ, Kenemans P. Postmenopausal hormone therapy: impact on menopause-related symptoms, chronic disease and quality of life. Drugs. 2004;64(8):821-36.

25. Hunter M. The Women's Health Questionnarie: a measure of mid-aged women's perceptions of their emotional and physical health. Psychol Health. 1992;7(1):45-54.

26. Silva-Filho CR, Baracat EC, Haidar MA, Ferraz MB. Climacteric symptoms and quality of life: validity of women's health questionnaire. Rev Saude Publica. 2005;39(3):333-9.

27. Snaith RP, Bridge GWK, Hamilton M. The Leeds's scales for the self-assessment of anxiety and depression.Br J Psychiatry. 1976; 128:156-65

28. Hamilton M. The assessment of anxiety states by rating. $\mathrm{Br} \mathrm{J}$ Med Psychol. 1959;32(1):50-5.

29. Hamilton M. Development of a rating scale for primary depressive illness. Br J Soc Clin Psychol. 1967;6(4):278-96.

30. Hunter MS. Psychological and somatic experience of the menopause: a prospective study [corrected]. Psychosom Med. 1990; 52(3): 357-67. Erratum in Psychosom Med 1990;52(4):410.

31. Slaven L, Lee C. A Cross-sectional Survey of Menopausal Status, Symptoms and Psychological Distress in a Community Sample of Australian Women. J Health Psychol. 1998;3(1):1 17-23.

32. Wiklund I, Berg G, HammarM, Karlberg J, Lingren R, Sandin NK. Longterm effect of transdermal hormonal therapy on aspects of quality of life in postmenopausal women. Maturitas .1992; 14(3): 225-36.

33. Shin H, Shin HS. Measurement of quality of life in menopausal women: A systematic review. West J Nurs Res published online 25 March 2011 . [internet] Disponível em: http://wjn.sagepub. com/content/early/2011/03/30/0193945911402848

34. Hu XH, Markson LE, Lipton RB, Stewart WF, Berger ML. Burden of migraine in the United States: disability and economic costs. Arch Intern Med. 1999; 159:813-8.

35. National Center for Health Statistics. Vital and Health Statistics of the United States. Dept of Health, Education, and Welfare. Advance data. Hyattsville, MD: National Center for Health Statistics, 1979. PHS Publication No. 53. Disponível em: http:// www.cdc.gov/nchs/products/pubs/pubd/nvsr/53/53-prehtm

36. Celentano DD, Stewart WF, Lipton RB, Reed ML. Medication use and disability among migraineurs: a national probability sample. Headache. 1992;32(5):223-8.

37. Fry J. Profiles of Disease. Edinburgh: Churchill Livingstone, 1996:45-56.

38. Bigal ME, Bordini CA, Speciali JG. Headache in an emergency room. São Paulo Med J. 2000;1 18:58-62.

39. Lipton RB, Stewart WF, Scher Al. Epidemiology and economic impact of migraine. Curr Med Res Opin. 2001;17(suppl 1):4S-12S.

40. Lipton RB, Stewart WF, Diamond S, Diamond ML, Reed M. Prevalence and burden of migraine in the United States: data from the American Migraine Study II. Headache. 2001 ; 41 (7):646-57.

41. Dahlöf C, Bouchard J, Cortelli P, et al. A multinational investigation of the impact of subcutaneous sumatriptan. II. Health-related quality of life. Pharmacoeconomics. 1997; 1 1 (suppl 1):24 -34

42. Santanello NC, Polis AB, Hartmaier SL, Kramer MS, Block GA, Silberstein SD. Improvement in migraine-specific quality of life in a clinical trial of rizatriptan. Cephalalgia. 1997;17(8):867- 2.

43. American Headache Society, 2007 MIDAS (The Migraine Disability Assessment Test). [Internet] Disponível em: http:// www.americanheadachesociety.org/
44. Stewart WF, Lipton RB, Dowson AJ, Sawyer J. 2001. Development and testing of the Migraine Disability Assessment (MIDAS) Questionnaire to assess headache-related disability. Neurology. 56:S20-S28.

45. Winner P, Ricalde O, Le Force B, Saper J, Margul B. A double blind study of sub-cutaneous dihydroergotamine vs subcutaneous sumatriptan in the treatment of acute migraine. Arch Neurol. 1996;53(2): 180-4. Comment in Arch Neurol. 1996;53(12): $1215-6$.

46. Martin VT, Behbehani M. Ovarian hormones and migraine headache: understanding mechanisms and pathogenesis--part 2. Headache. 2006; 46(3):365-86.

47. Brandes JL. The influence of estrogen on migraine: a systematic review. JAMA. 2006; 295(15): 1824-30.

48. Lipton RB, Stewart WF. Migraine in the United States: a review of epidemiology and health care use. Neurology. 1993; 43(6 Suppl 3):S6- 10.

49. Waters WE, O'Connor PJ. Epidemiology of headache and migraine in women. J Neurol Neurosurg Psychiatry. 1971 ;34(2): 148-53.

50. Melhado EM, Maciel JA Jr, Guerreiro CA. Headache during gestation: evaluation of 1101 women. Can J Neurol Sci. 2007; 34(2): 187-92.

51. Kornstein SG, Parker AJ. Menstrual migraines: Etiology, treatment, and relationship to premenstrual syndrome. Curr Opin Obstet Gynecol. 1997;9(3):154-9.

52. Neri I, Granella F, Nappi R, Manzoni GC, Facchinetti F, Genazzani AR. Characteristics of headache at menopause: a clinico-epidemiologic study. Maturitas 1993; 17(1):31-7.

53. Mattsson P. Hormonal factors in migraine: a population-based study of women aged 40 to 74 years. Headache. 2003;43(1): 27-35.

54. Ciciarelli MC. Cefaléia e ciclo hormonal. In Cefaléias (Speciali, JG. \& Silva, WF. Editores), 2002.

55. Wang SJ, Fuh JL, Lu SR, Juang KD, Wang PH. Migraine prevalence during menopausal transition. Headache. 2003; 43(5):470-8. Comment in Headache. 2004;44(1):106; author reply 106.

56. Calhoun AH. Migraine and menopause. Headache 2004; 44(1): 106; author reply 106. Comment on Headache. 2003 May;43(5):470-8.

57. Somerville BW. Estrogen-withdrawal migraine. II. Attempted prophylaxis by continuous estradiol administration. Neurology. 1975;25(3):245-50.

58. Somerville BW. Estrogen-withdrawal migraine. I. Duration of exposure required and attempted prophylaxis by premenstrual estrogen administration. Neurology. 1975; 25(3):239-44.

59. Misakian AL, Langer RD, Bensenor IM, Cook NR, Manson JE, Buring JE, et al. Postmenopausal hormone therapy and migraine headache. J Womens Health (Larchmt) 2003; 12(10): 1027-36.

Correspondência

Sidraiton Sálvio Alves de Melo Filho Rua Mamanguape, 455/103 - Boa Viagem 51020-250 - Recife, PE, Brasil e-mail: sidraitonfilho@hotmail.com

Recebido: 8/1/2012

Aceito: 28/2/2012 